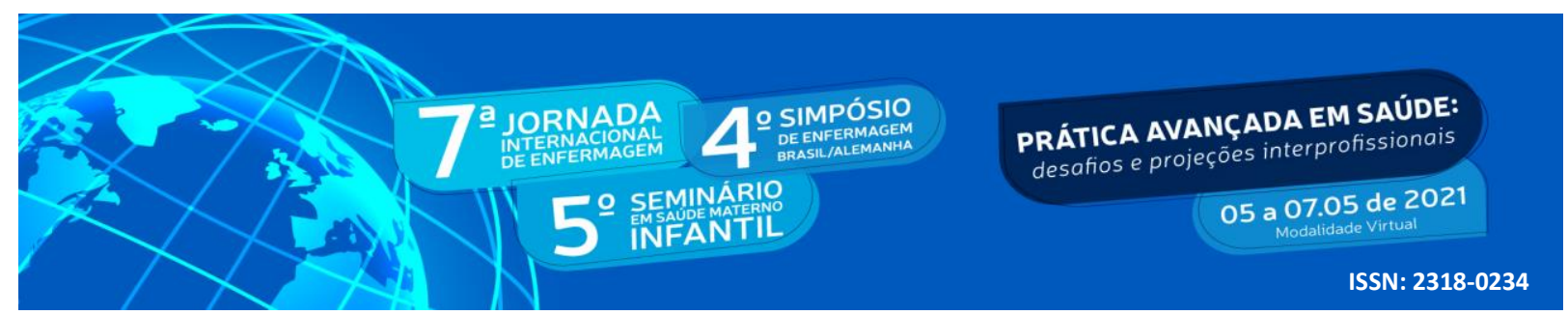

DOI: http://doi.org/10.48195/jie2021-043

\title{
ATIVIDADE EDUCATIVA SOBRE PRÁTICAS INTEGRATIVAS E COMPLEMENTARES ${ }^{1}$
}

\section{Larissa Pereira Righi da Silva²; Leandro da Silva de Medeiros ${ }^{3}$; Giovana Luiza Rossato $^{4}$; Luiza Fortes Lamberty ${ }^{5}$; Rosiane Filipin Rangel ${ }^{6}$}

\begin{abstract}
RESUMO
Objetivo: Relatar a experiência de acadêmicos de enfermagem acerca de uma atividade educativa sobre práticas integrativas e complementares. Método: Trata-se de um relato de experiência, desenvolvido por estudantes de Enfermagem a partir da vivência na disciplina de Espiritualidade e Saúde, no segundo semestre de 2019. Resultados: Verificou-se que as atividades educativas, sobretudo, no ambiente acadêmico, contribuem para a quebra de paradigmas no que tange a temática das práticas integrativas e complementares e dessa forma possibilitam visualizar o ser humano na integralidade. Conclusão: Considera-se que as práticas integrativas e complementares geram impactos significativos no cuidado à saúde, portanto, as atividades educativas orientam os saberes de profissionais e estudantes para o cuidado humanizado.
\end{abstract}

Palavras-chave: Enfermagem; Humanização da Assistência; Atenção à Saúde; Terapias Complementares.

\begin{abstract}
Objective: To report the experience of nursing students about an educational activity on integrative and complementary practices. Method: This is an experience report, developed by nursing students from their experience in the discipline of Spirituality and Health, in the second semester of 2019. Results: It was found that educational activities, especially in the academic environment, contribute to break paradigms with regard to the theme of integrative and complementary practices and thus make it possible to view the human being in its entirety. Conclusion: It is considered that integrative and complementary practices generate significant impacts on health care, therefore, educational activities guide the knowledge of professionals and students towards humanized care.
\end{abstract}

\footnotetext{
${ }^{1}$ Trabalho desenvolvido na disciplina de Espiritualidade e Saúde, do curso de graduação em Enfermagem, da Universidade Franciscana- UFN.

${ }^{2}$ Estudante do Curso de Enfermagem da Universidade Franciscana - UFN. Santa Maria, Rio Grande do Sul, Brasil. E-mail: larissarighi89@gmail.com

${ }^{3}$ Estudante do Curso de Enfermagem da Universidade Franciscana- UFN. Santa Maria, Rio Grande do Sul, Brasil. E-mail: leandro.medeiros@ufn.edu.br

${ }^{4}$ Estudante do Curso de Enfermagem da Universidade Franciscana- UFN. Santa Maria, Rio Grande do Sul, Brasil. E-mail: rossatogiovana@gmail.com

${ }^{5}$ Estudante do Curso de Enfermagem da Universidade Franciscana- UFN. Santa Maria, Rio Grande do Sul, Brasil. E-mail: luizaflamberty@gmail.com

${ }^{6}$ Orientadora. Doutora em Enfermagem. Docente do Curso de Graduação em Enfermagem e do Mestrado Profissional em Saúde Materno Infantil da Universidade Franciscana. Santa Maria, Rio Grande do Sul, Brasil. Email: rosianerangel@yahoo.com.br
} 


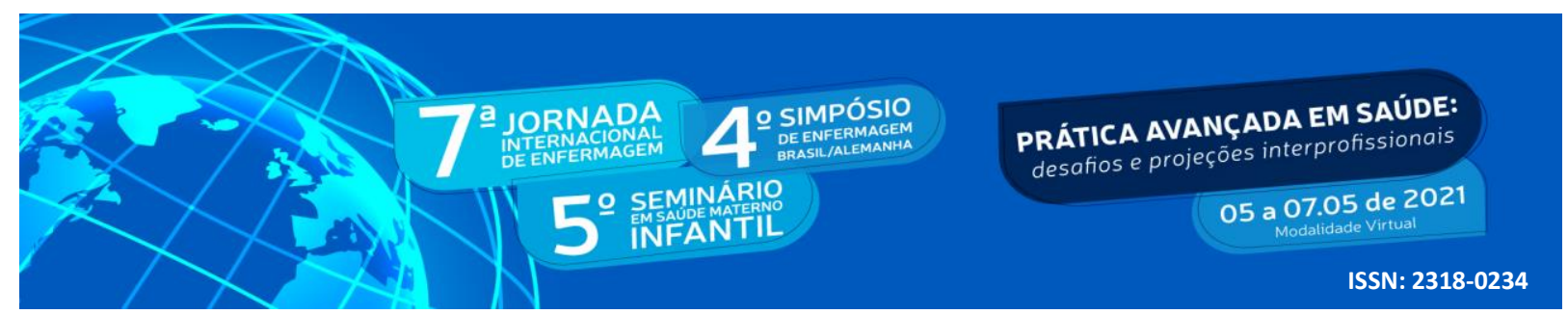

Key Words: Nursing; Humanization of Assistance; Health Care ; Complementary Therapies.

\section{INTRODUÇÃO}

As Práticas Integrativas e Complementares (PICS) são parte de uma política governamental desde os anos 70, mas foram aprovadas pelo Ministério da Saúde, como Política Nacional de Práticas Integrativas e Complementares em Saúde (PNPIC), por meio da Portaria GM/MS n ${ }^{\circ}$ 971, em 3 de maio de 2006. Com isso foram recebendo visibilidade devido os questionamentos do cuidado hegemônico, ou seja, o paciente que não é compreendido na sua singularidade, mas apenas no cuidado centrado no diagnóstico com tratamento medicamentoso (BRASIL, 2019).

Na PNPIC são ofertadas, por meio do Sistema Único de Saúde (SUS), 29 PICS aos cidadãos. Essas terapias são complementares e tem por objetivo cuidar de forma subjetiva e singular a pessoa, evidenciando-se que é uma prática integrativa, no qual traz inúmeros benefícios (BRASIL, 2019).

O crescimento da população, o aumento da expectativa de vida, a multiplicação de técnicas, disciplinas e novas especialidades médicas que proliferam de modo alucinante são ao mesmo tempo a riqueza e o drama maior do setor saúde, especialmente por afetarem as relações comunicativas, a ação coordenada dos novos conhecimentos e a integração do conjunto imenso de informações que se produz (JÚNIOR, 2016).

Os profissionais que utilizam as PICS como prática de assistência ao cuidado, não realizam simplesmente porque aprenderam outra técnica de saúde e desejam aplicá-la, mas são movidos pela vontade de reformular uma identidade de cuidado habitual. Trata-se de mostrar que existem alternativas capazes de fazer a diferença e se tornar parte de um processo de melhoria e evolução do paciente além dos diferentes modos de promover saúde, menos onerosos e mais aptos a cuidar do ser humano em sua totalidade (JÚNIOR, 2016).

Apesar da polêmica e da diversidade de conceitos e nomenclaturas, o tema atribui algumas características comuns às Medicinas Tradicionais/Complementares e Alternativas, mesmo que não mutáveis para todo o conjunto e nem para todas as situações ou praticantes, haja vista sua diversidade: a procura de cura e equilíbrio por meio de ações que reforçam ou induzem uma resposta natural do organismo; um princípio holístico, com enfoque integral dos 


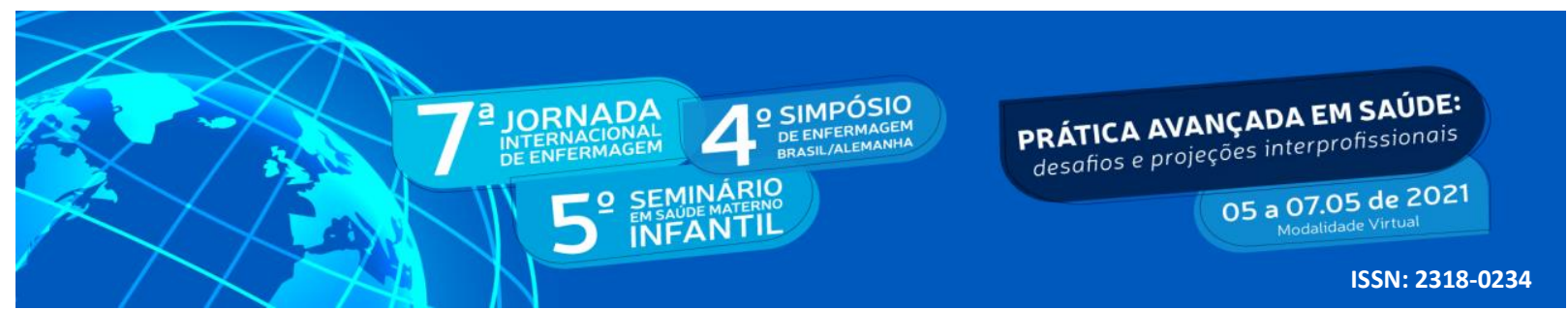

problemas de saúde e da vida; a busca do equilíbrio entre a mente, o corpo e seu entorno; a ênfase na saúde em lugar da doença. Várias delas têm como objetivo a existência de uma "dinâmica vital", energia, espírito, "sopro", que está além da noção de funcionalidade fisiológica e orgânica e que pode ser estimulada (BRAGA, 2019).

Portanto, o objetivo deste estudo consiste em relatar a experiência construída em sala para reconhecer e construir estratégias de cuidado singular de acordo com Organização Mundial da Saúde (OMS) sobre Medicina Tradicional, PNPIC, atenção integral e humanizada ao indivíduo; assim descreve-se a prática do Feng Shui que consiste no uso científico das Terapias integrativas/Complementares, campos de força, meditação, saúde e espiritualidade, terapia floral, aromaterapia, cromoterapia, fitoterapia, reiki, toque terapêutico e harmonização de ambientes para que o usuário tenha uma entrega espiritual e espontânea para a sua melhora em todas as dimensões.

\section{OBJETIVO}

Relatar a experiência de acadêmicos de enfermagem acerca de uma atividade educativa sobre práticas integrativas e complementares.

\section{METODOLOGIA}

Trata-se de um relato de experiência, desenvolvido por discentes de enfermagem a partir de vivências teórico-práticas realizadas na disciplina optativa Espiritualidade e Saúde que compõem a grade curricular do terceiro semestre do curso de graduação em enfermagem. O estudo desenvolveu-se ao longo do segundo semestre do ano de 2019, sob orientação da professora responsável pela disciplina.

$\mathrm{O}$ relato de experiência se traduz em um texto acadêmico que descreve precisamente uma dada experiência que possa contribuir de forma relevante para sua área de atuação profissional. Caracteriza-se por uma vivência profissional (acadêmica) considerada importante para a formação dos futuros profissionais da área da enfermagem, em seu meio acadêmico (FERREIRA, 2019).

Para fundamentar o estudo, por meio da literatura, foram realizadas buscas de artigos na Biblioteca Virtual em Saúde (BVS), utilizando os descritores: "Práticas" and "Terapias 


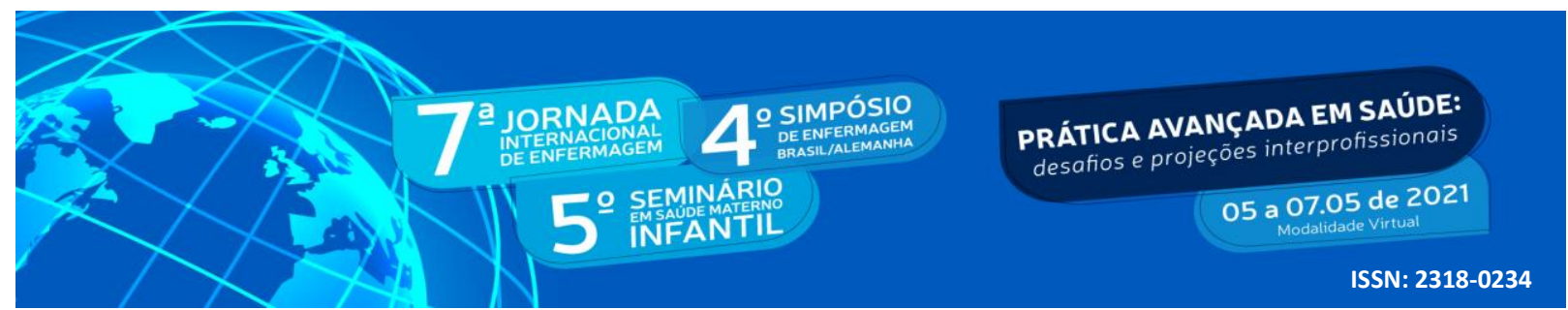

Complementares" and "Saúde" and "Enfermagem". Os dados recolhidos foram analisados e vinculados com a experiência dos acadêmicos que cursaram a disciplina conjuntamente das orientações da professora responsável.

Ressalta-se que a atividade educativa foi realizada por meio de uma palestra expositiva-dialogada por uma terapeuta, na qual, discorreu sobre o assunto "Feng Shui" para a turma de 30 alunos.

\section{RESULTADOS E DISCUSSÃO}

A terapeuta, discorreu sobre a importância das práticas integrativas e complementares em saúde, e também sobre a sua experiência com florais de bach, reiki, entre outros. Segundo, Mendes et al (2019), as PICS visam aumentar a qualidade de vida do paciente, através de práticas que estimulem o bem-estar físico e mental, assim como redução de danos de agravos, promovendo um melhor ambiente de tratamento.

Assim, em roda de conversa junto com os estudantes da disciplina, a terapeuta holística falou sobre algumas práticas para serem usadas contra a ansiedade, outras para momentos de concentração e memorização. O momento mais discutido foi sobre ansiedade, pois acomete muitos estudantes, repercutindo negativamente, por vezes, nos afazeres da academia.

A ansiedade é considerada um transtorno, na qual os pacientes possuem sinais como estresse, fobias e agonias. Diante disso, as PICS podem ser utilizadas como auxiliares no tratamento e no dia a dia de pessoas (GOIATÁ, et al 2016). Além disso, é um sentimento que acomete muitos graduandos, visto que, no decorrer da vida acadêmica, precisamos lidar com situações estressoras que geram ansiedade, exigindo recursos psicológicos e emocionais para o manejo das exigências e das adversidades durante a formação (REIS; MIRANDA; FREITAS, 2019).

Algumas práticas podem ser adotadas para a diminuição da ansiedade como meditação e reiki, segundo a terapeuta. Além disso, outras terapias podem fazer parte no tratamento da ansiedade e no plano de cuidados dos enfermeiros. A professora responsável pela disciplina enfatizou a importância da assistência em saúde não ser focado somente na enfermidade, mas também no bem estar mental, físico, social e espiritual de cada ser humano, 


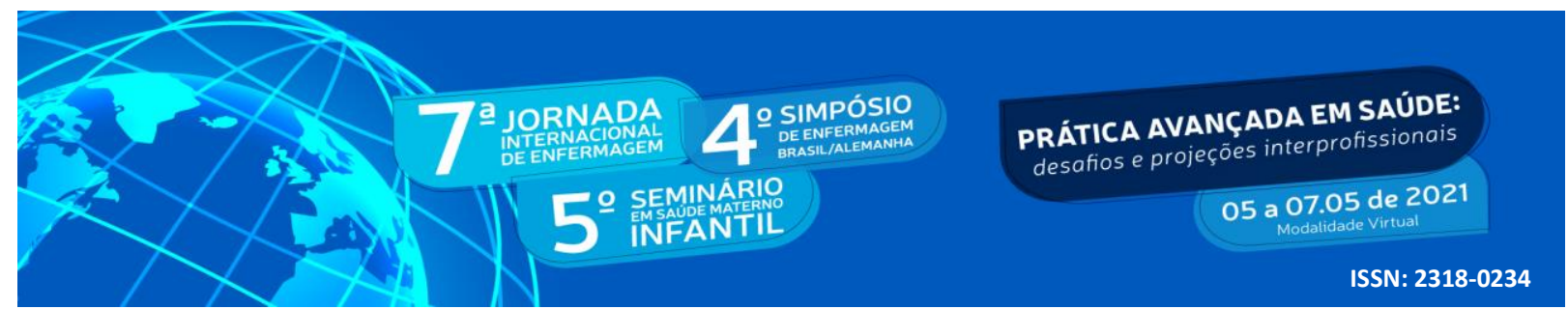

sempre buscando respeito às suas crenças e valores.

Ao decorrer da palestra, a terapeuta holística argumentou sobre alguns florais, entre eles os florais de bach, que são considerados PICS e sua fundamentação apresenta grande cunho espiritual, entendendo que as pessoas possuem uma alma que é o seu eu real e divino, e que, através de uma relação harmoniosa entre alma e personalidade, tem-se como resultado a saúde (ARRUDA, 2012).

Quando se discute sobre as PICS, surgem dúvidas de alguns profissionais de saúde se podem ou não aplicarem na assistência do cuidado. O Conselho Federal de Enfermagem (COFEN) em março de 2018, por meio de sua página oficial, demonstrou apoio às iniciativas de implementação das práticas integrativas no Brasil, considerando que a adoção de novas práticas na PNPIC demonstram um avanço do modelo de saúde alinhados aos princípios do SUS.

Umas das práticas recomendada pela terapeuta é o Reiki, sendo um sistema de cura através da imposição das mãos, utilizado para o tratamento do corpo físico, atuando na parte mental, emocional e espiritual. É marcado pela autocura, autoconhecimento, liberdade de escolha e de consciência, trazendo benefícios que vão além do corpo físico e age na causa dos sintomas. É um tipo de terapia oferecida a indivíduos em situação de saúde e de doença, ela aumenta a energia vital e fortalece o sistema imunológico (FREITAG et al 2014).

Ao final da palestra, a terapeuta pediu para que formassem um círculo, próximo ao som. Pois mediou uma meditação em torno de 10 minutos e todos puderam sentir um pouco do trabalho da mesma para realização no cuidado ao ser humano. Observou-se a expressão e relato dos alunos acerca do bem-estar e da paz que estavam sentindo com a fala e prática realizada. A meditação é uma prática que desenvolve bem-estar físico e mental e, com isso, redução de efeitos psicossomáticos negativos (ESPER; GNATTA; SILVA, 2016).

\section{CONCLUSÃO}

Considera-de a importância de disciplinas como a de Espiritualidade e saúde nas grades curriculares dos cursos da área da saúde, entre outros. Enquanto discentes, percebe-se como é relevante o conhecimento acerca das PICS, pois trazem benefícios tanto para o paciente, quanto para quem está cuidando-o, ou seja, os profissionais da saúde. Além disso, 


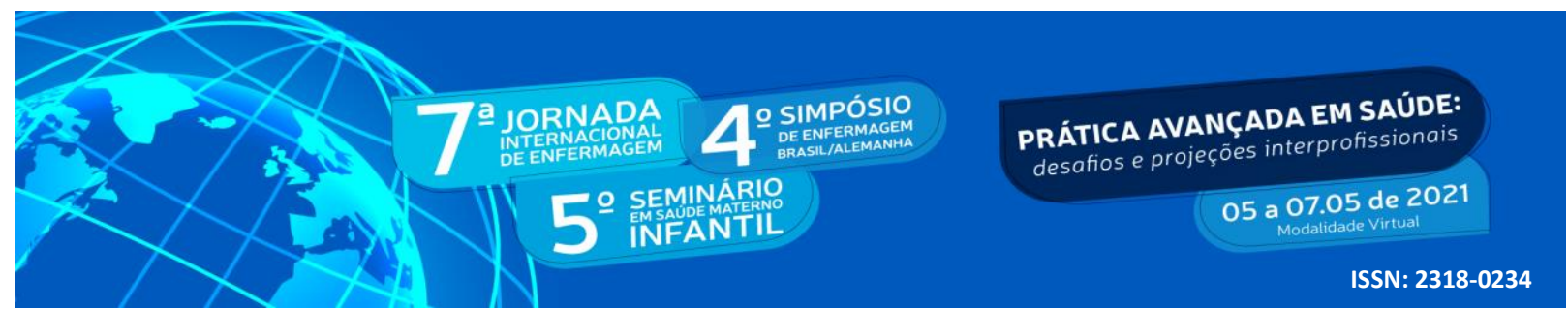

promove a autonomia dos pacientes, por vezes redução de custos e a possibilidade de cuidar na integralidade humana considerando todas as dimensões.

Por fim, destaca-se que a enfermagem, assim como demais profissionais que compõem a equipe de saúde, de visualizarem as PICS como um modelo de cuidado a ser ensinado e praticado no ambiente do cuidado.

\section{REFERÊNCIAS}

ARRUDA, APCCBN. Efetividade dos florais de Bach no bem estar espiritual de estudantes universitários: ensaio clínico randomizado duplo cego. Botucatu-SP. Pág.130, 2012.

BRAGA, H. H. M. Práticas Integrativas e Complementares e Educação Permanente em Saúde: implicação na Atenção Primária à Saúde de Minas Gerais. 2019. Dissertação (Mestrado em Enfermagem) - Escola de Enfermagem da Universidade Federal de Minas Gerais, Minas Gerais, 2019.

BRASIL. Ministério da Saúde. Práticas Integrativas e Complementares (PICS): quais são e para que servem. Disponível em: http://saude.gov.br/saude-de-a-z/praticas-integrativas-ecomplementares\#historico. Acesso em: 10 dez. 2019.

CONSELHO FEDERAL DE ENFERMAGEM. Cofen manifesta apoio as práticas integrativas e complementares. Brasília; 2018. Disponível em: http://www.cofen.gov.br/cofenmanifesta-apoio-as-praticasintegrativasecomplementares_61201.html. Acesso em: 15 dez 2019.

ESPER M.V., GNATTA J.R., SILVA M.J.P. Caracterização da produção científica sobre meditação na área da saúde e na Enfermagem: revisão de literatura. Cad Naturologia Terapias Complementares. v. 5, n.8, pág. 39-46, 2016.

FERREIRA, E. C.. Relatos de Experiência. Projeto de Pesquisa de conclusão de Especialização em Atenção ao Paciente Crítico: Urgência, Emergência e UTI (UNINTER). 2019.

FREITAG VL, et al.. Benefícios do Reiki em população idosa com dor crônica. Texto Contexto Enferm. v.23, n.4, pág. 1032-1040, 2014.

GOYATÁ S.L.T.; et al. Effects from acupuncture in treating anxiety: integrative review. Rev Bras Enferm. v. 69, n. 3, pág. 564-571, 2016.

JÚNIOR, E.T. Práticas integrativas e complementares em saúde, uma nova eficácia para o SUS: subtítulo do artigo. Estudos Avançados: subtítulo da revista, São Paulo, v. 30, n. 86, p. 99-112, mar./2016.

MENDES, DS et al. Benefícios das práticas integrativas e complementares no cuidado de 


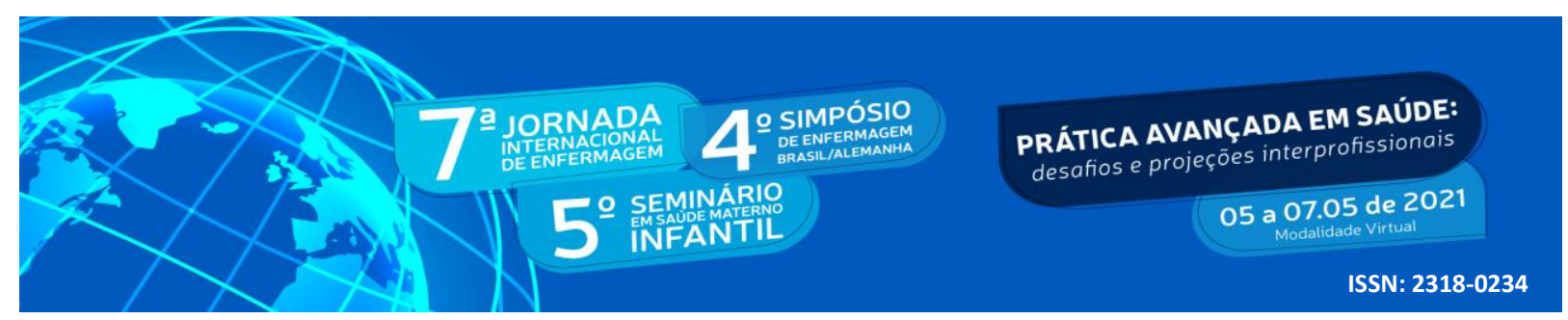

enfermagem. Journal Health NPEPS. v. 4, n.1, pág.302-318, jan-jun 2019.

REIS, C. F.; MIRANDA, G. J.; FREITAS, S. C. Ansiedade e desempenho acadêmico: um estudo com alunos de ciências contábeis. Advances in Scientific and Applied Accounting, [S. l.], v. 10, n. 3, p. 319-333, 2017. Disponível em: https://asaa.anpcont.org.br/index.php/asaa/article/view/356. Acesso em: 15 dez. 2019. 